\title{
SUPERIOR APPROACH TO THE LEFT MAIN CORONARY ARTERY FOR SURGICAL ANGIOPLASTY
}

Kiyoyuki Eishi, MD, Hiroaki Sasaki, MD, Kiyoharu Nakano, MD, Yoshio Kosakai, MD, Fumitaka Isobe, MD, Yoshikado Sasako, MD, and Junjiro Kobayashi, MD, Osaka, Japan

The three reported surgical approaches to the left main coronary arterial trunk (LMT) are anterior, ${ }^{1}$ posterior, ${ }^{2}$ and lateral. ${ }^{3}$ The anterior and posterior approaches offer the best access to the LMT orifice. The anterior approach

From the Division of Cardiovascular Surgery, National Cardiovascular Center, Osaka, Japan.

Received for publication June 25, 1996; accepted for publication Sept. 24, 1996.

Address for reprints: Kiyoyuki Eishi, MD, Division of Cardiovascular surgery, National Cardiovascular Center of Japan, 5-7-1

Fujishiro-dai, Suita, Osaka, 565 Japan.

J Thorac Cardiovasc Surg 1997;113:609-11

Copyright (C) 1997 by Mosby-Year Book, Inc.

$0022-5223 / 97 \$ 5.00+0 \quad \mathbf{1 2 / 5 4 / 7 8 1 7 6}$ is commonly used for ease of exposure of the LMT itself. However, kinking of an onlay patch at the junction of the LMT and the left posterolateral aortic wall may result in serious flow disturbances. ${ }^{1}$ An onlay patch placed by means of the posterior approach would rest flat from the LMT onto the right posterolateral aortic wall. However, exposure of the LMT from this angle is limited. To expose the whole LMT and avoid kinking of the patch, we developed a new approach using the cadaver heart. This "superior approach" was then successfully used clinically.

A 74-year-old man had angina pectoris for approximately 1 year. Coronary cineangiography revealed a $75 \%$ stenosis of the left coronary artery (LCA) at its origin. The remainder of the coronary arteries appeared normal. The LMT was long and had a large diameter, making angioplasty a viable treatment alternative (Fig. 1).
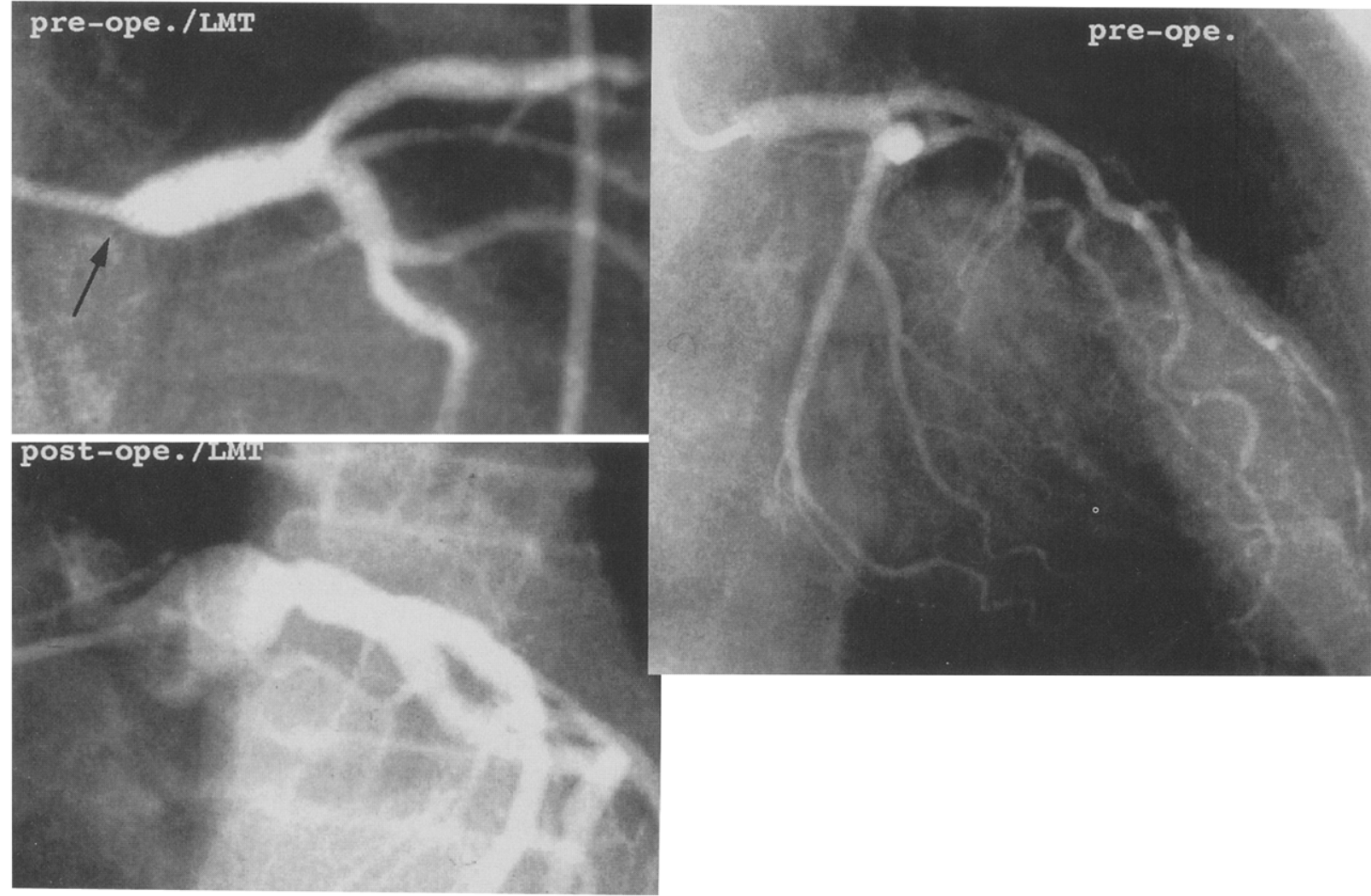

Fig. 1. Preoperative coronary angiograms revealed severe stenosis of the orifice of the LMT. There were no sclerotic changes in other branches. Postoperative coronary angiograms revealed an enlarged LMT orifice and excellent blood flow through the LMT. 

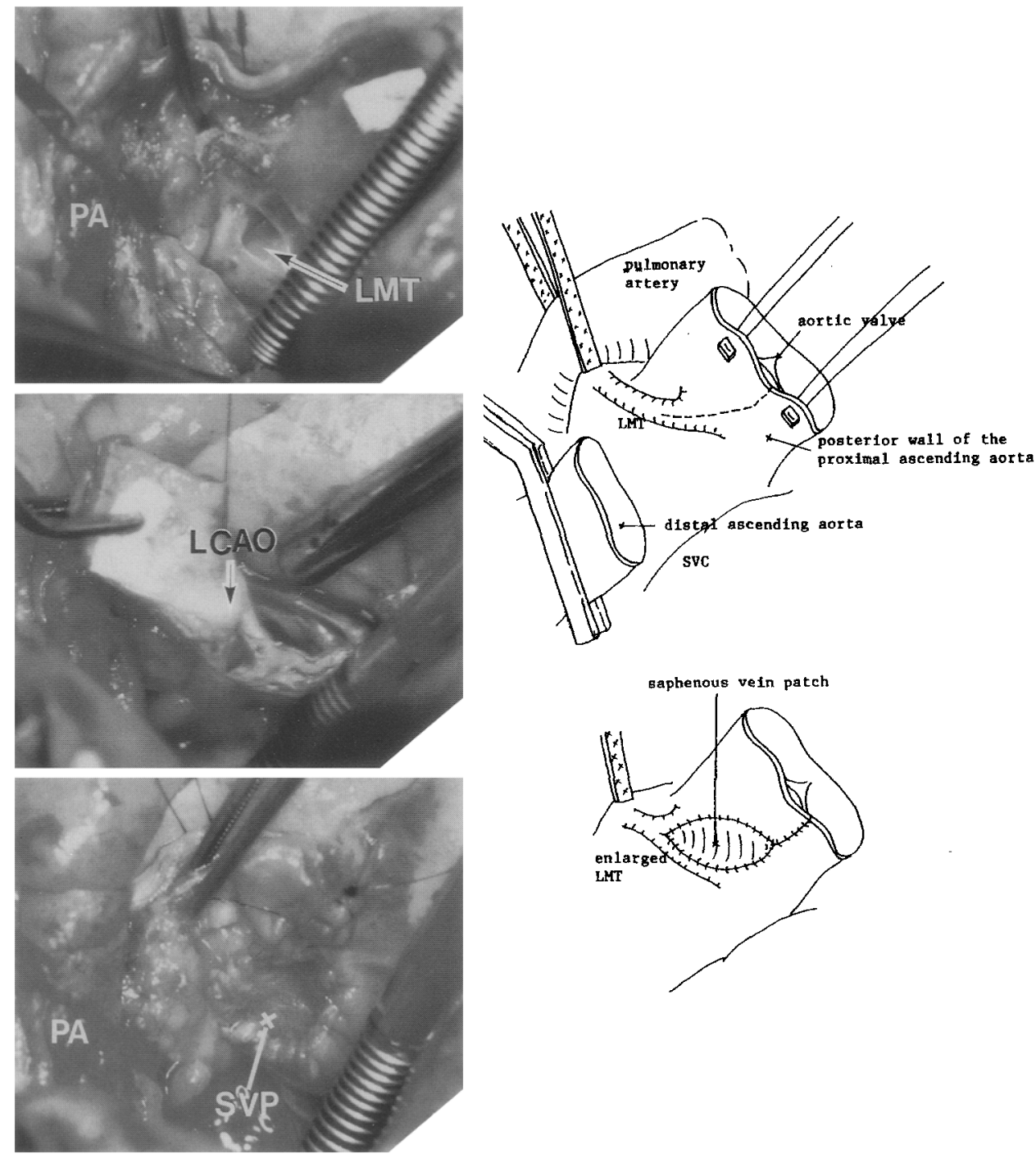

Fig. 2. The main pulmonary artery was retracted to its lateral side by an encircling tape and the posterior wall of the aorta was pulled inferiorly, after complete transection of the ascending aorta (top). The incision started near the left noncoronary commissure and slightly curved into the LMT (dotted line and middle photograph). Suturing of the saphenous vein patch began from the bottom of the LMT incision and continued upward. Patch angioplasty with a saphenous vein was completed (bottom). LMT, Left main trunk; $P A$, pulmonary artery; $L C A O$, left coronary artery orifice; $S V P$, saphenous vein patch; $S V C$, superior vena cava,

With the patient under neuroleptanalgesia and in the supine position, a median sternotomy was performed. A short segment of the saphenous vein was dissected and procured for the onlay patch. After extracorporeal circulation was begun, temporary cardioplegia was obtained with antegrade and retrograde infusion of St. Thomas' Hospital solution.

First, the ascending aorta was completely transected about $15 \mathrm{~mm}$ above the sinotubular junction. The posterior aortic wall was pulled inferiorly with two pairs of 4-0
Prolene stay sutures (Ethicon, Inc., Somerville, N.J.). The pulmonary trunk was retracted to the left with an encircling tape. By means of these maneuvers, the LMT and its junction with the posterior aortic wall were were exposed (Fig. 2, top). Examination of the orifice of the LCA from the lumen of the aorta revealed a diameter of $1.5 \mathrm{~mm}$. Another aortotomy was begun at the junction of the noncoronary and left coronary commissures and curved to the LCA orifice, entering the LMT along a straight line. The endothelium was atheromotous and partially calcified 
around the LCA orifice (Fig. 2, middle). This atheromatous material was removed very carefully, creating a wide orifice. Then a saphenous vein patch $(15 \times 10 \mathrm{~mm})$ was sutured with 7-0 Prolene sutures from the inferior border of the LMT incision up to the junction of the aortic wall, terminating $5 \mathrm{~mm}$ below the end of the aortotomy. After the saphenous vein patch was secured, the entire course of the LMT was carefully examined. The transected aorta was reconstructed with a continuous 4-0 Prolene suture (Fig. 2, bottom).

The patient was weaned from the pump without difficulty and transferred to the intensive care unit in good condition. There were no postoperative changes in the electrocardiogram. The recovery was uneventful, and the patient was discharged in good condition. Postoperative catheterization revealed a large LCA orifice and LMT (Fig. 1).

Surgical angioplasty of the LMT provides physiologic coronary perfusion and good late results without the difficulties associated with graft occlusion. ${ }^{4}$ Impaired LMT flow, however, may result in significant damage to the myocardium. ${ }^{5}$ Physiologic reconstruction after exposure of the entire LMT is necessary to provide the best flow through this vessel. The "superior approach" de- scribed here is an effective way to gain wide exposure of the LMT for surgical manipulation.

\section{REFERENCES}

1. Dion R, Verhelst R, Matta A, Rousseau M, Goenen M, Chalant C. Surgical angioplasty of the left main coronary artery. J Thorac Cardiovasc Surg 1990;99:241-50.

2. Hitchcock JF, Robles de Medina EO, Jambroes G. Angioplasty of the left main coronary artery for isolated left main coronary artery disease. J Thorac Cardiovasc Surg 1983;85: 880-4.

3. Sabiston DC, Ebert PA, Friesinger GC, et al. Proximal endarterectomy: arterial reconstruction for coronary occlusion at the aortic origin. Arch Surg 1965;91:758-64.

4. Sen RC, Hitter E, Ranquin R, Cawwelaert V, Lieber S, Vanden-Branden F. Surgical coronary angioplasty for left main vasospasm. Am Heart J 1995;129:399-400.

5. Favaloro RG, Effler DB, Groves LK, Sheldon WC, Shirey EK Sones FM. Severe segmental obstruction of the left main coronary and its division: surgical treatment by the saphenous vein graft technique. J Thorac Cardiovasc Surg 1970;60:46982

\title{
WHOLE BLOOD HEPARIN CONCENTRATION MEASUREMENTS BY AUTOMATED PROTAMINE TITRATION AGREE WITH PLASMA ANTI-XA MEASUREMENTS
}

\author{
G. J. Despotis, MD, J. H. Joist, MD, PhD, L. T. Goodnough, MD, S. A. Santoro, MD, PhD, and E. Spitznagel, PhD, \\ St. Louis, Mo.
}

Findings recently reported by our group indicated that point-of-care monitoring of heparin concentration by an automated protamine sulfate titration (PST) method (Hepcon HMS, Medtronic Hemotec, Inc., Englewood, Colo.) can reduce excessive microvascular bleeding and blood component transfusion. ${ }^{1}$ That study was preceded by another evaluation in which we found a high degree of correlation between heparin concentration determined by

From the Departments of Anesthesiology, Internal Medicine, Pathology, and Surgery, Washington University School of Medicine, St. Louis, Mo., and the Departments of Internal Medicine and Pathology, St. Louis University School of Medicine, St. Louis, Mo.

Received for publication Sept. 23, 1996; accepted for publication Oct. 22, 1996.

Address for reprints: George Despotis, MD, Division of Cardiothoracic Anesthesiology, Department of Anesthesiology, Box 8054, Washington University School of Medicine, 660 South Euclid Ave., St. Louis, MO 63110.

J Thorac Cardiovase Surg 1997;113:611-3

Copyright () 1997 by Mosby-Year Book, Inc.

$0022-5223 / 97 \$ 5.00+0 \quad \mathbf{1 2 / 5 4 / 7 8 7 6 8}$ plasma anti-Xa and whole blood automated PST. ${ }^{2}$ Hardy and colleagues ${ }^{3}$ recently published a comparison of heparin measurements by Hepcon automated PST and a laboratory-based anti-Xa chromogenic assay in a relatively small number of patients undergoing cardiac operation with the use of cardiopulmonary bypass (CPB). In contrast to our observations of a strong linear relationship between whole blood PST and plasma anti-Xa measurements, ${ }^{2}$ these investigators found unacceptably high variability between the two heparin concentration measurements. In explaining this discrepancy, Hardy and colleagues ${ }^{3}$ suggested that our data analysis was limited and potentially misleading because we did not use bias analysis. We therefore reanalyzed our data with a bias analysis method as used by that group.

Plasma anti-Xa and whole blood PST measurements (duplicate) on blood specimens obtained at sequential operative times ( 4 to 6 per patient) from 62 patients undergoing cardiac operations were reanalyzed. ${ }^{2}$ Whole blood (WB) PST heparin values were converted to plasma equivalent values (PE) using concurrently measured hematocrit (Hct) values with the following formula: PE WB PST $=($ WB PST $\times 100 /[100-\mathrm{Hct}])$. The mean difference or bias between plasma anti-Xa 